\title{
Theoretical diagnostic and prediction of physical properties of quaternary InGaAsP compound using artificial neural networks optimized by the Levenberg Maquardt algorithm
}

\author{
Amal Tarbi ${ }^{1}$ - El Houssine Atmani ${ }^{1} \cdot$ Mohammed Amine Sellam ${ }^{2} \cdot$ Meriem Lougdali $^{1}$. \\ Youssef El Kouari ${ }^{1} \cdot$ Anna Migalska-Zalas $^{3}$
}

Received: 2 June 2018 / Accepted: 29 June 2018 / Published online: 9 July 2018

(C) The Author(s) 2018

\begin{abstract}
The quaternaries $\operatorname{In}_{1-x} G a_{x} A s_{y} P_{1-y}$ are the main promising elements for the fabrication of optoelectronic devices. The adjustment of their physical parameters is assumed by the change of the molar fraction $x$ and $y$. These parameters can be affected by the variation of temperature and pressure. To make the theoretical diagnosis of these materials, it is fundamental to know the energy gap ' $\boldsymbol{E}_{\boldsymbol{g}}$ ' and the lattice parameter ' $a$ ', over a wide range of chemical compositions $0 \leq x \leq 0.47$ and $0 \leq y \leq 1$, at different temperatures and pressures. We show that by using the Artificial Neural Network method optimized by the Levenberg Maquardt algorithm ANN-LM, it is possible to obtain results very close to the experiment. The scatter plot and error calculation show that the ANN-LM model provides more accurate values of the lattice parameter than those calculated by Vegard's law. On the other hand, the energy gap values $E g(x, y, T)$ estimated, using the ANN-LM model, proved to be close to the experimental values that those calculated by the empirical equations. In addition, the ANN-LM method allowed us to estimate with great accuracy the values of the energy gap at different temperatures and pressures $\operatorname{Eg}(P, T)$. Our work provides crucial information on the physical properties of the quaternary without the use of approximations, and without taking into account the hypothesis of a perfect agreement between InGaAsP and $I n P$ substrate.
\end{abstract}

Keywords Quaternary InGaAsP · Lattice parameters · Bandgap energy · Pression and temperature · Artificial neural network · Levenberg Maquardt Algorithm · Vegard's law

Amal Tarbi

amal_tarbi@yahoo.fr

$\triangle$ Anna Migalska-Zalas

aniazal@op.pl

1 Laboratory of Condensed Matter and Renewable Energy, Faculty of Sciences and Technology, University Hassan II of Casablanca, BP 146, Mohammedia, Morocco

2 Laboratory of Engineering Sciences, ESTEM Center for Technological Innovation and Scientific Research (CITRS), Casablanca, Morocco

3 Institute of Physics, Faculty of Mathematics and Natural Science, Jan Dlugosz University in Czestochowa, Al. Armii Krajowej 13/15, 42-200 Czestochowa, Poland 


\section{Introduction}

The $I I I-V$ ternary and quaternary semiconducting materials have attracted a great scientific and technological research interest last decade, thanks to their unique and exceptional electrical and optical properties missing in Silicon. Among these properties one can note high mobility of electrons, which found in InGaAs componds, make them promising candidates to manufacture MOSFETs components (Xuan et al. 2008). Also, the possibility to tune the gap energy value in these materials provides a large panel of optoelectronic applications, such as photodetectors, long wavelength semiconductor lasers (Agrawal 1986), light-emitting diodes (LEDs) (Tarbi and Mani 2015), and optic fiber for high-speed data communications (Fashinger et al. 1994) devices. Further arguments concern the development of supplementary electronic heterostructures (Akiba et al. 1979).

InGaAsP quaternary system we suggest to study here is an $I I I-V$ semiconducting material, which crystalizes in the bulk zinc-blende (ZB) crystal structure. Its chemical synthesis can be performed by using epitaxial crystal growth techniques, on the surface of an $I n P$ substrat, over a wide range of chemical $x$ and $y$ compositions (Seifert and Runge 2016; Manasevit 1968). Physical properties of the InGaAsP alloys such as the lattice parameter and the energy gap can be changed by modifying many control parameters such as chemical composition ratio $x$ and $y$ (Vurgaftman et al. 2001), temperature and even pressure (Degheidy and Elkenany 2015). The alloy prepared on the InP substrate covers a range of energies gap ranging from 0.75 to $1.35 \mathrm{eV}$, whilst, it takes values between 1.42 and $1.9 \mathrm{eV}$ when it prepared on GaAs substrate (Houston 1981).

This shows their great strength which lies in the almost unlimited number of possible alloys between the four elements $G a, A s$, In and $P$ (Adachi 1982). In particular conditions, one can observe associated with a good matching between lattice parameters of both materials ( $\operatorname{In} G a A s P / \operatorname{InP})$, a direct band-gap. However, most theoretical calculations of these physical properties are computed by Virtual Crystal Approximation (VCA) and the Vegard's law (Shim et al. 2000).

This research aims to assume an ANN-LM estimation model for the prediction and the representation of the physical behavior of the quaternary $\operatorname{In}_{1-x} G a_{x} A s_{y} P_{1-y}$ $(0 \leq x \leq 0.47 ; 0 \leq \mathrm{y} \leq 1)$ with a high performance compared to the empirical equations used. To show their abilities to model physical phenomena, the predicted results using ANN-LM, and the values computed by equations based on the adoption of interpolation will be compared by the experimental values of the lattice parameters and of the energy gap, by the error indicators MSE, RMSE, and $R^{2}$.

Beyond the state of the art, we presume the above method can be used in several applications. In fact, it offers a complementary method to provide clues about problems concerning complex physical phenomena for existed material. It can be even used for studding and developing new materials. Thus, this pre-manufacturing possibility provides an optimal parameter needed with a low cost and time. In the particular case of photovoltaic cells, the predictions provided by the ANN-LM estimation model method, can be helpful to unveil and expect the temperature-dependent energygap fluctuations in the InGaAsP/InP material. In fact, the so called thermalization loss process, as shown in Fig. 1 below, is responsible of heat transfer to lattice and thus the temperature of the network and disturbs $E_{g}(T)$. 


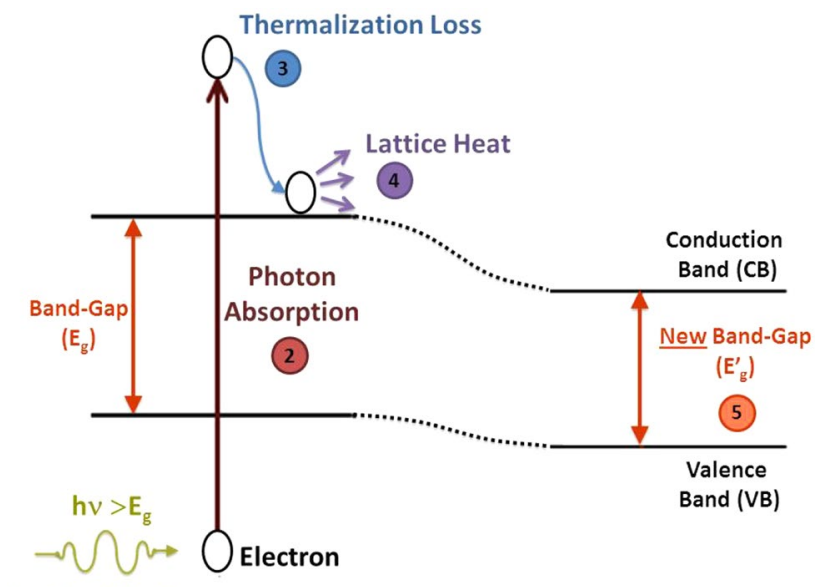

Incident Photon

(1)

Fig. 1 Schematic view of the thermalization loss effect on the band-gap. The different steps are numbered according to time order

\section{Theoretical analysis}

\subsection{Lattice parameters of quaternary material $/ n_{1-x} G a_{x} A s_{y} P_{1-y} / I n P$ : Vergard's law}

The quaternaries $A_{x} B_{1-x} C_{y} D_{1-y}$ consist of two group III elements and two group $V$ elements. To calculate their band parameters, the interpolation approximation (Glisson et al. 1978) is used. It is given by (Krijn 1991):

$$
G_{A B C D}^{\prime \prime}(x, y)=\frac{x(x-1)\left[(1-y) G_{A B D}^{\prime}(x)+y G_{A B C}^{\prime}(x)\right]+y(1-y)\left[x G_{A C D}^{\prime}(y)+(1-x) G_{B C D}^{\prime}(y)\right]}{x(1-x)+y(1-y)}
$$

The band parameter of the ternary $\mathrm{A}_{1-\mathrm{x}} \mathrm{B}_{\mathrm{x}} \mathrm{C}$ can be expressed as (Krijn 1991):

$$
G_{A B C}^{\prime}(x)=(1-x) G_{A C}+x G_{B C}-x(1-x) C_{A B C}
$$

With $G_{A C}$ and $G_{B C}$ are the values of the binary $A C$ and $B C$ respectively, and $C_{A B C}$ is the appropriate bowing.

The Vegard's law is an approximation derived from the linear combination of the lattice constants of the GaAs, GaP, InAs and InP binary compounds, which constitute the quaternary material $I n_{1-x} G a_{x} A s_{y} P_{1-y}$ (Mei 2007), it is given by the following relation:

$$
a(\mathrm{x}, \mathrm{y})=\mathrm{xy} a(\mathrm{GaAs})+\mathrm{x}(1-\mathrm{y}) a(\mathrm{GaP})+(1-\mathrm{x}) \mathrm{y} a(\operatorname{InAs})+(1-\mathrm{x})(1-\mathrm{y}) a(\operatorname{InP})
$$

With: $a(G a A s)=5.6536 \AA, a(G a P)=5.4512 \AA, a(\operatorname{InAs})=6.0590 \AA$, and $a(\operatorname{InP})=$ $5.8696 \AA$, the relationship becomes:

$$
a(x, y)=0.1894 y-0.4184 x+0.0130 x y+5.8696
$$

The lattice mismatch can be calculated by (Vurgaftman et al. 2001):

$$
f=\frac{a(x, y)-a(\operatorname{In} P)}{a_{\operatorname{In} P}}
$$


In order to obtain a perfect match between the material $\operatorname{InGaAs} P$ and the substrate $\operatorname{In} P$, the condition below must be imposed.

$$
a(x, y)=a(\operatorname{In} P)
$$

With $a(x, y)$ represents the lattice parameter of the material $I n_{1-x} G a_{x} A s_{y} P_{1-y}$, and $a(\operatorname{In} P)$ indicates the lattice parameter of the InP substrate. In the absence of a mismatch, we consider $y \approx 2.2 x$ (Vurgaftman et al. 2001; Houston 1981). Equation (4) becomes a single dimension:

$$
a(y)=5.8696-0.0008 y+0.0059 y^{2}
$$

With $a$ is measured in $\AA$. The lattice parameter may undergo a change due to the increase in temperature, according to the Adachi relation (Degheidy and Elkenany 2015):

$$
a(T)=a(300 \mathrm{~K})\left[1+\alpha_{t h}(1-300 \mathrm{~K})\right]
$$

where: $\alpha_{t h}$ is linear thermal expansion coefficient (Table 1). The pressure can also change the lattice parameter, according to the relationship given by Adachi (Degheidy and Elkenany 2015):

$$
a(p)=a(p=0)\left[1+\left(\frac{B^{\prime}}{B}\right) p\right]^{\frac{-1}{3 B^{\prime}}}
$$

where $a(p)$ is the lattice parameter at different pressures, $B$ is the bulk modulus and $B \prime$ is the pressure derivative of the bulk modulus for the associated binary semiconductors (see Table 1).

\subsection{Energy gap of the quaternary $\ln _{1-x} G a_{x} A s_{y} P_{1-y}$}

The Electroreflectance (ER) allows to determine the energy gap of the quaternary alloy In $G a A s P$ adapted to InP. The measurement of the photoluminescence spectrum also makes it possible to specify the energy gap by measuring the peak energy spectrum (Yamazoe et al. 1981). The difference between the two methods is that the (ER) spectrum is not sensitive to the effects of impurities, defects and errors in the alloy (Yamazoe et al. 1981).

There are models that have been developed for the prediction and computation of the band structures based on the pseudopotential method (Jones and Lettington 1969). The

Table 1 Varshni's parameter and hydrostatic pressure of the binary semiconductors which constitute the InGaAsP quaternary (Degheidy and Elkenany 2015)

\begin{tabular}{lllll}
\hline & GaAs & GaP & InP & InAs \\
\hline$b\left(10^{-3} \mathrm{eV} / \mathrm{Kbar}\right)$ & 11.5 & -1.3 & 8.2 & 11.4 \\
$c\left(10^{-5} \mathrm{eV} / \mathrm{Kbar}^{2}\right)$ & -24.5 & 0 & 0 & 0 \\
$\alpha\left(10^{-4} \mathrm{~K}^{-2}\right)$ & 5.41 & 5.8 & 4.5 & 3.07 \\
$\beta(\mathrm{K})$ & 204 & 387 & 335 & 191 \\
$\alpha_{t h}\left(10^{-6} \mathrm{~K}^{-1}\right)$ & 6.03 & 4.65 & 4.56 & 5 \\
$\mathrm{~B}(\mathrm{Gpa})$ & 75.5 & 88.19 & 72.3 & 57.9 \\
$\mathrm{~B}$ & 4.49 & 4.5 & 4.49 & 4.79 \\
\hline
\end{tabular}

Bold values indicate the number of neurons that gave the smallest RMSE 
theoretical approaches that explain the data remain modest. Numerous articles and books have dealt with the methods of computation of the heterostructure and bulk band structures (Vurgaftman et al. 2001; Yu and Cardona 1996). The energy gap as a function of the compositions of $x$ and $y$ is not linear, the modeling in this case becomes complex. The simplification of this relationship has been achieved by geometric methods based on the use of the four ternary boundaries (Nahory et al. 1978). The energy gap at room temperature (300 K) is in the form:

$$
E_{g}(x, y)=\left(1.35+0.668 x-1.17 y+0.758 x^{2}+0.18 y^{2}-0.069 x y-0.322 x^{2} y+0.03 x y^{2}\right) \mathrm{eV}
$$

This relationship shows a strong inclination with respect to the experimental measurements (Nahory et al. 1978; Nakajima et al. 1978). The solution for finding a very good agreement is to use the empirical fit of the curve drawn by the experimental points. The equation of adjustment is given by the following relation:

$$
\mathrm{E}_{\mathrm{g}}(\mathrm{y})=\left(1.35-0.72 \mathrm{y}+0.12 \mathrm{y}^{2}\right) \mathrm{eV} \text { at }(T=300 \mathrm{~K})
$$

The model has been improved by adding coefficient represents the effective bowing parameter. The relation becomes (Yamazoe et al. 1980):

$$
\mathrm{E}_{\mathrm{g}}(\mathrm{y})=1.35 .(1-\mathrm{y})+0.75 \mathrm{y}-\mathrm{C}_{\mathrm{e}} \cdot \mathrm{y} \cdot(1-\mathrm{y})
$$

With: $C_{e}=0.138$. Operation of the material at a higher or lower temperature than ambient temperature affects its electrical properties and performance. As a result, Model 11 becomes far from accurate. The energy gap of the $\operatorname{InGaAsP}$ material at $0 \mathrm{~K}$ is given by the relation (Waqas et al. 2016):

$$
\mathrm{E}_{\mathrm{g}}(\mathrm{y})=\left(1.421-0.72 \mathrm{y}+0.12 \mathrm{y}^{2}\right) \mathrm{eV} \text { at }(T=0 \mathrm{~K})
$$

Taking into account that the energy gap of the $\operatorname{In} P$ varies between $1.35 \mathrm{eV}$ and $1.421 \mathrm{eV}$ when the temperature varies from 300 to $0 \mathrm{~K}$. The difference between the energy gap Eqs. (11) and (13) is reflected in the first term which represents the energy gap of the InP. The coefficients of composition remain the same. On the other hand, the semi-empirical law of Varshni presents the equation which predicts the forbidden energy as a function of temperature in the semiconductor (Varshni 1967):

$$
E_{g}(T)=E_{g 0}-\frac{\alpha T^{2}}{T+\beta}(\text { Kelvin })
$$

With: $E_{g 0}$ is the bandgap energy at temperature $0 \mathrm{~K} . \alpha$ and $\beta$ are adjustment constants. The energy gap as a function of temperature and composition is deduced from relation (13) and (14):

$$
E_{g}(T, y)=\left[\left(E_{g 0}-\frac{\alpha T^{2}}{T+\beta}\right)-0.72 y+0.12 y^{2}\right](\mathrm{eV})
$$

The good estimation of the energy gap also makes it possible to calculate the refractive index of the alloy $\operatorname{In}_{1-x} G a_{x} A s_{y} P_{1-y}$ at $T=300 \mathrm{~K}$. According to the semi-empirical modified single oscillator model (Fiedler and Schlachetzki 1987), the relation can be written as:

$$
n^{2}=1+\frac{E_{d}}{E_{0}}+\frac{E_{d}}{E_{0}^{3}} E^{2}+\frac{\eta}{\pi} E^{4} \ln \left(\frac{2 E_{0}^{2}-E^{2}-E_{g}^{2}}{E_{g}^{2}-E^{2}}\right)
$$




$$
\text { With: } \quad \eta=\frac{\pi E_{d}}{2 E_{0}^{3}\left(E_{0}^{2}-E_{g}^{2}\right)}
$$

$E_{0}$ is the energy of the single-effect oscillator, $E_{d}$ is the dispersion energy, and $E=h v$ is the photon energy. The energies $E_{0}$ and $E_{d}$ are calculated with the interpolation method (Nahory et al. 1978). At ambient temperature, these parameters are in the form of approximations (Waqas et al. 2016):

$$
\begin{gathered}
E_{0}(y)=3.391-1.652 y+0.863 y^{2}-0.123 y^{3} \\
E_{d}(y)=28.91-9.278 y+5.626 y^{2}
\end{gathered}
$$

The direct and indirect energy gap of the material subjected to the pressure $E_{g}^{d, i d}(T, p)$ at the points of high symmetry $\Gamma, X$ and $L$ is obtained by using the following empirical relation (Degheidy and Elkenany 2015):

$$
E_{g}^{d, i d}(T, p)=E_{g}(T, p=0)^{d, i d}+b p+c p^{2}
$$

where: $b$ and $c$ are the pressure coefficients, cited in Table 1 (Degheidy and Elkenany 2015).

\subsection{Artificial neural network optimized by the Levenberg-Marquardt algorithm (ANN-LM)}

The Artificial Neural Network ANN is a strong mathematical tool for modeling and prediction, it is a black box based on learning from examples. It is characterized by the generalization of the process in a flexible way and with a great speed. It is capable of generating a particular, nonlinear and complex function between inputs and outputs by adjusting the biases and weights that represent the network's digital knowledge (Mekki et al. 2016). ANN modeling does not provide a tangible model that ensures the physical understanding of the underlying process in the material.

The Multilayer Perceptron MLP is a feed forward network that guarantees multi-layer transmission via neurons (Tarbi et al. 2017) as shown in Fig. 2. The processing of the

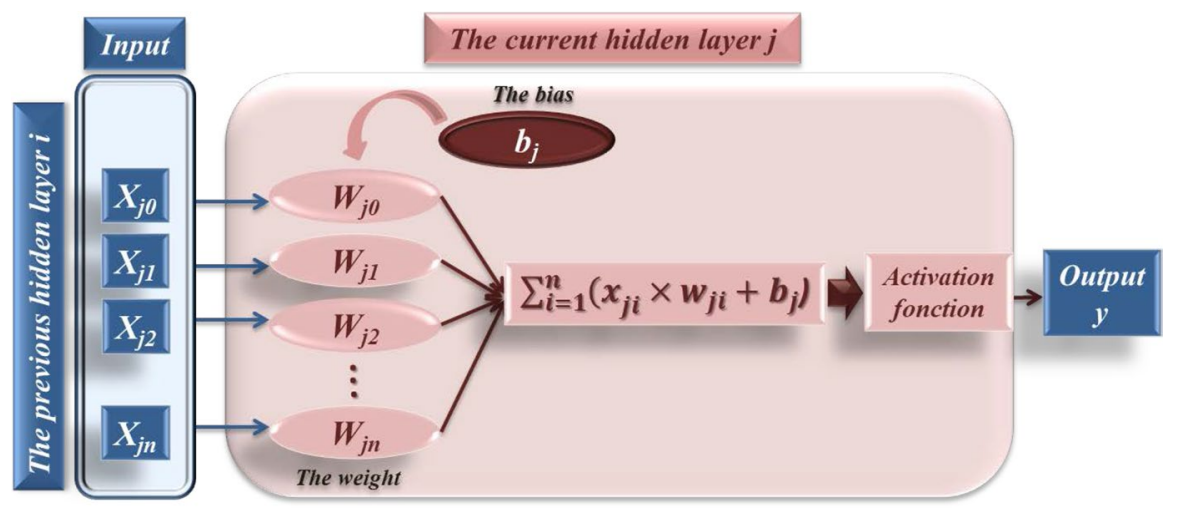

Fig. 2 Method of processing information by the node 
information of each node has been represented in Fig. 2 and calculated by the mathematical Eq. (21). The output of this node serves as input of another node of the following layer (Kumari et al. 2016; Dkhichi et al. 2016):

$$
y=f\left(\sum_{i=1}^{n}\left(x_{j i} \times w_{j i}+b_{j}\right)\right)
$$

With: $y$ is the output of the current node of the hidden layer $j . n$ is the number of the inputs of the current layer, i.e. the number of nodes in the previous hidden layer, $x_{j i}$ an input of the current hidden layer $j$ from the previous layer $i, \mathrm{w}_{\mathrm{ji}}$ the weight that joins layer $j$ and the layer $i, f$ is the activation function. The LogSig, TanSig and Purelin activations functions are defined by:

$$
\begin{gathered}
\operatorname{LogSig}(x)=\frac{1}{1+e^{-x}} \\
\operatorname{TanSig}(x)=\frac{e^{x}-e^{-x}}{e^{x}+e^{-x}} \\
\operatorname{Purelin}(x)=x
\end{gathered}
$$

Experimental measurements are used for the formation of ANN-LM. The back-propagation (BP) algorithm used to minimize the error function is the Levenberg-Marquardt iterative learning algorithm, due to its precision and fast convergence (Dkhichi et al. 2014). The performance function such as the mean square error (MSE) stops the training process when it takes values smaller than the predefined threshold. The formation also stops when it has reached the desired number of epochs (Afram et al. 2017). The variance indicators used in this work for the evaluation of the quality of the prediction are the RMSE (Geyikçi et al. 2012), and the determination coefficient $R$-square (Chen et al. 2017) defines respectively:

$$
\begin{gathered}
R M S E=\left(\frac{1}{n} \sum_{i=1}^{n}\left(y_{\text {pred }}-y_{\text {exp }}\right)^{2}\right)^{1 / 2} \\
R^{2}=1-\left(\frac{\sum_{i=1}^{n}\left(y_{\text {pred_ }-} y_{\text {exp }}\right)^{2}}{\sum_{i=1}^{n}\left(y_{\text {avg,exp }} y_{\text {exp }}\right)^{2}}\right)
\end{gathered}
$$

where $n$ is the number of targets or the number of experimental responses; $y_{\text {pred }}$ and $y_{\text {exp }}$ are the predicted and target data, respectively, and $y_{\text {avg,exp }}$ is the average of the target values. In this work, the physical properties of the quaternary alloy $\operatorname{In}_{1-x} G a_{x} A s_{y} P_{1-y}$ where $x$ and $y$ denote the composition of Gallium and Arsenic respectively, are studied using the ANN$L M$ model. 
Table 2 Procedure for selecting the optimal transfer function for predicting the lattice parameter by the ANN method
Table 3 Procedure for selecting the optimal number of neurons with the best transfer function TangSig/Purlin for ANN training

\begin{tabular}{|c|c|c|c|c|}
\hline $\begin{array}{l}\text { Number } \\
\text { of layers }\end{array}$ & Train function & $\begin{array}{l}\text { Number of } \\
\text { neurons }\end{array}$ & Transfer function & RMSE \\
\hline \multirow[t]{9}{*}{2} & Trainlm & 10 & LogSig/LogSig & 0.0067 \\
\hline & & & LogSig/Purelin & 0.0043 \\
\hline & & & LogSig/TangSig & 0.0045 \\
\hline & & & Purelin/LogSig & 0.0062 \\
\hline & & & Purelin/Purelin & 0.0061 \\
\hline & & & Purelin/TangSig & 0.0075 \\
\hline & & & TangSig/LogSig & 0.0049 \\
\hline & & & TangSig/Purelin & 0.0025 \\
\hline & & & TangSig/TangSig & 0.0049 \\
\hline
\end{tabular}

Bold values indicate the transfer function that gave the smallest RMSE

\begin{tabular}{|c|c|c|c|c|}
\hline $\begin{array}{l}\text { Number } \\
\text { of layers }\end{array}$ & Train function & Transfer function & $\begin{array}{l}\text { Number of } \\
\text { neurons }\end{array}$ & RMSE \\
\hline \multirow[t]{15}{*}{2} & Trainlm & Tangsig/Purelin & 1 & 0.0053 \\
\hline & & & 2 & 0.0029 \\
\hline & & & 4 & 0.0014 \\
\hline & & & 6 & 0.0025 \\
\hline & & & 8 & 0.0026 \\
\hline & & & 10 & 0.0112 \\
\hline & & & 12 & 0.0025 \\
\hline & & & 14 & 0.0023 \\
\hline & & & 16 & 0.0018 \\
\hline & & & 18 & 0.0029 \\
\hline & & & 20 & 0.0069 \\
\hline & & & 22 & 0.0015 \\
\hline & & & 24 & 0.006 \\
\hline & & & 26 & 0.0073 \\
\hline & & & 28 & 0.0034 \\
\hline
\end{tabular}

Bold values indicate the number of neurons that gave the smallest RMSE

\section{Result and discussion}

\subsection{Estimation of the lattice parameter of the quaternary $\ln _{1-x} G a_{x} A s_{y} P_{1-y} / \operatorname{In} P$}

$A N N-L M$ models were used to predict the lattice parameter ' $a$ '. Some networks are better adjusted than others. The procedure for choosing the optimal neural network is to analyze their performance using RMSE, as presented in Tables 2 and 3. The optimal artificial neural network is shown in Fig. 3, its architecture contains four layers: The first is the 'input layer', the last is the 'output layer', and between these two layers, there are two 'hidden layers'. In our case, two hidden layers give more accurate results than a single layer .

By default, it separates the experimental data into three categories: $70 \%$ of the data for the training, $15 \%$ were used for the test, and $15 \%$ for validation. The model is optimized by 


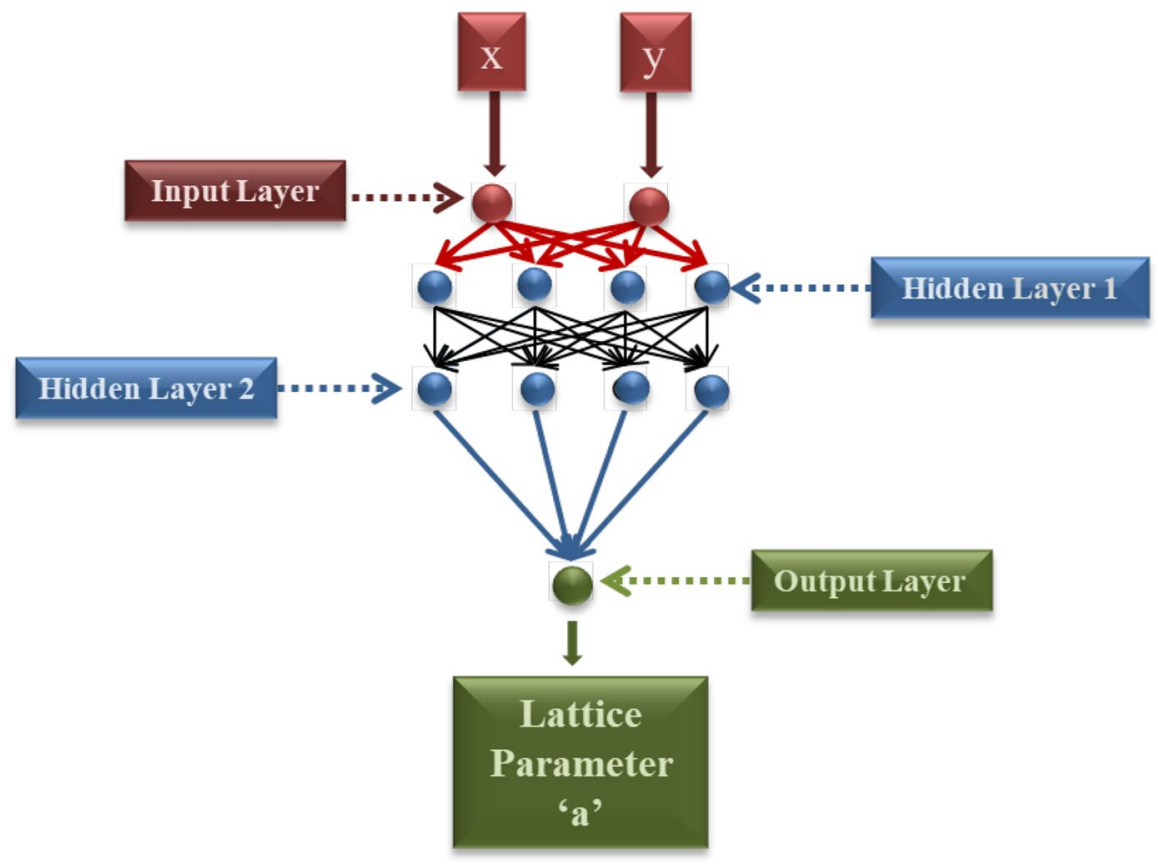

Fig. 3 Topology of the multilayer feedforward artificial neural network for the identification and the prediction of the lattice parameter ' $\mathrm{a}$ '. $\mathrm{x}$ and $\mathrm{y}$ are the chemical compositions of Gallium and Arsenic respectively

the Levenberg Maquardt algorithm 'Trainlm'. Neural networks with several hidden layers (more than three layers) lead to the problem of 'over fitting', generally they do not give optimum performance of prediction (Aish et al. 2015; Gazzaz et al. 2012).

The execution of various iterations makes it possible to specify the optimal and desirable scenario, since there is no theoretical rule for determining the number of neurons and the hidden layers of the neural network. Table 2 shows the transfer function choice methodology: The number of neurons was temporarily fixed at 10, the transfer function was changed by calculating the RMSE each time. The optimal function found is TangSig/Purelin, because it marks the smallest $R M S E$ of among the 9 combinations. The second step is to choose the number of neurons optimized: We did the simulation using the optimal function TangSig/Purelin, changing the number of neurons regularly. The smallest RMSE and the largest $R^{2}$ correspond to 4 neurons $\left(R M S E_{A N N}=0.0014\right.$ and $\left.R_{A N N}^{2}=95.84 \%\right)$.

We notice from the Fig. 4 that the values $\left(a_{A N N}\right)$ are very close to the values $\left(a_{\text {exp }}\right)$. The difference between the values $\left(a_{\text {exp }}\right)$, and the values estimated by the Vegard' law $\left(a_{\text {vegard }}\right)$ becomes acute when the composition of Arsenic is equal to 0.531. The RMSE and $R^{2}$ reach

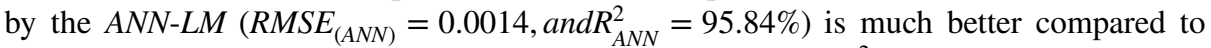
those completed by the Vegard's law $\left(R M S E_{\text {vegard }}=0.006\right.$, and $\left.R_{\text {vegard }}^{2}=17.73 \%\right)$. The horizontal dotted line corresponds to the lattice parameter of the InP substrate $a(\operatorname{InP})$.

when the values of the lattice parameters $a(x, y)$ converge to the value $a(\operatorname{In} P)$ (i.e. the horizontal line), we can say that there is an agreement between the quaternary $I n_{1-x} G a_{x} A s_{y} P_{1-y}$ and the InP substrate.

The figure shows the graphical representation of the correlation between the estimated data by the $A N N-L M$ method and by the Vegard's law (Fig. 5) and the measured 


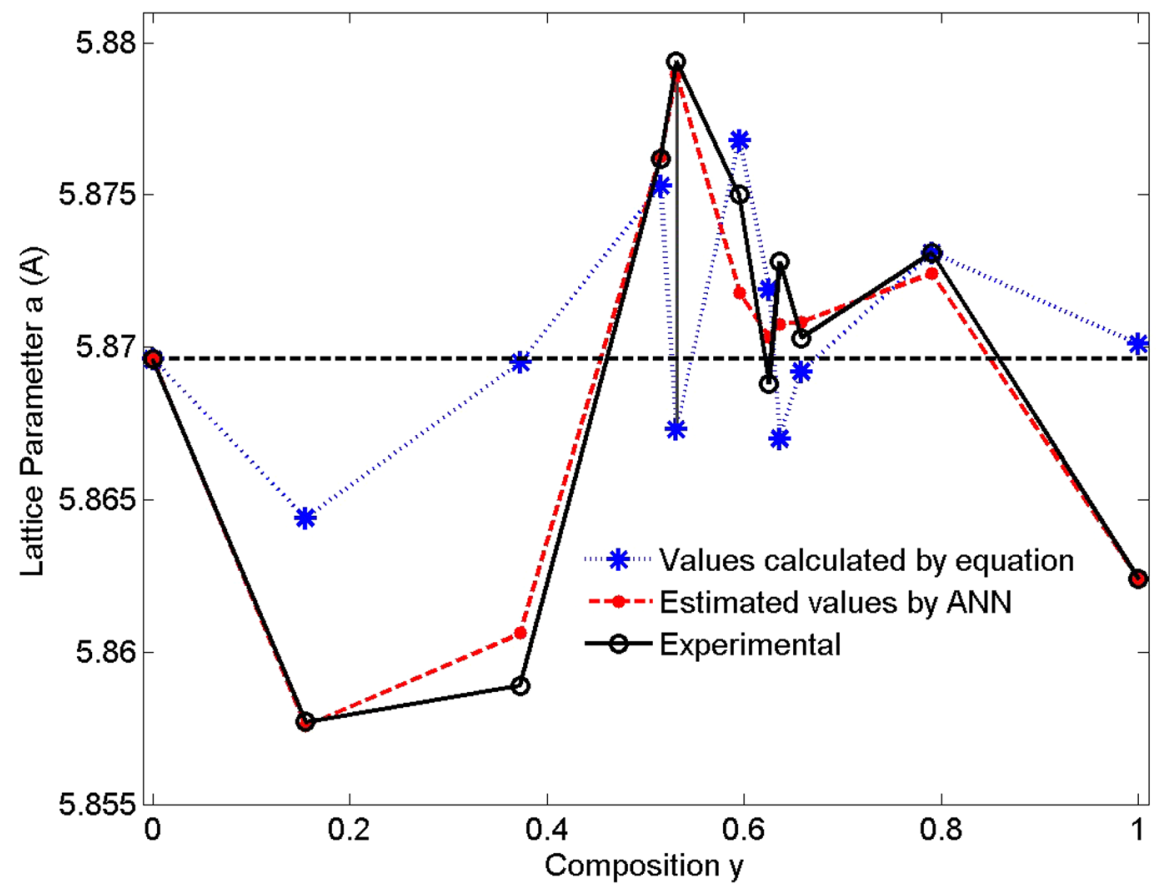

Fig. 4 Comparison between the experimental measurements $\left(\mathrm{a}_{\exp }\right)$, the data calculated by the Vegard's law $\left(\mathrm{a}_{\text {vegard }}\right)$ described by Eq. (4), and data predicted by ANN-LM $\left(\mathrm{a}_{\mathrm{ANN}}\right)$

data of the lattice parameter $a\left(\operatorname{In}_{1-x} G a_{x} A s_{y} P_{1-y}\right)$. It shows that in the case of the use of Vegard's law, the correlation coefficient is very far from 1 . Contrariwise, when estimating by $A N N-L M$ model, the slope becomes close to 1 . These results show that there is a perfect correlation between the values estimated by the $A N N-L M$ model and the targets, and they confirm the robustness of the $A N N-L M$ to predict values close to experimental values, and this, in the case of the absence or existence of the accordance between the quaternary and the substrate, that is to say, whatever the value of $x$ and $y$.

On the other hand, the Fig. 6 shows the curve of the lattice parameter as a function of the composition $y$. It makes it possible to compare the experimental values, the values estimated by the ANN-LM model, and the values calculated by the Eq. (7) deduced on the basis of the hypothesis of the total agreement between the quaternary and the substrate (i.e. $y \approx 2.2 x$ ). The difference between the targets and the values calculated by Eq. (7) is given by an important $R M S E(R M S E=0.0071)$ larger than the RMSE calculated from the Eq. (4).

Thus, we notice that the use of the assumption of the total agreement, (i.e. $y \approx 2.2 x$ ) increases the error, because of the convergence of the values calculated by Eq. (7) to the horizontal dotted line as shown in Fig. 6. 


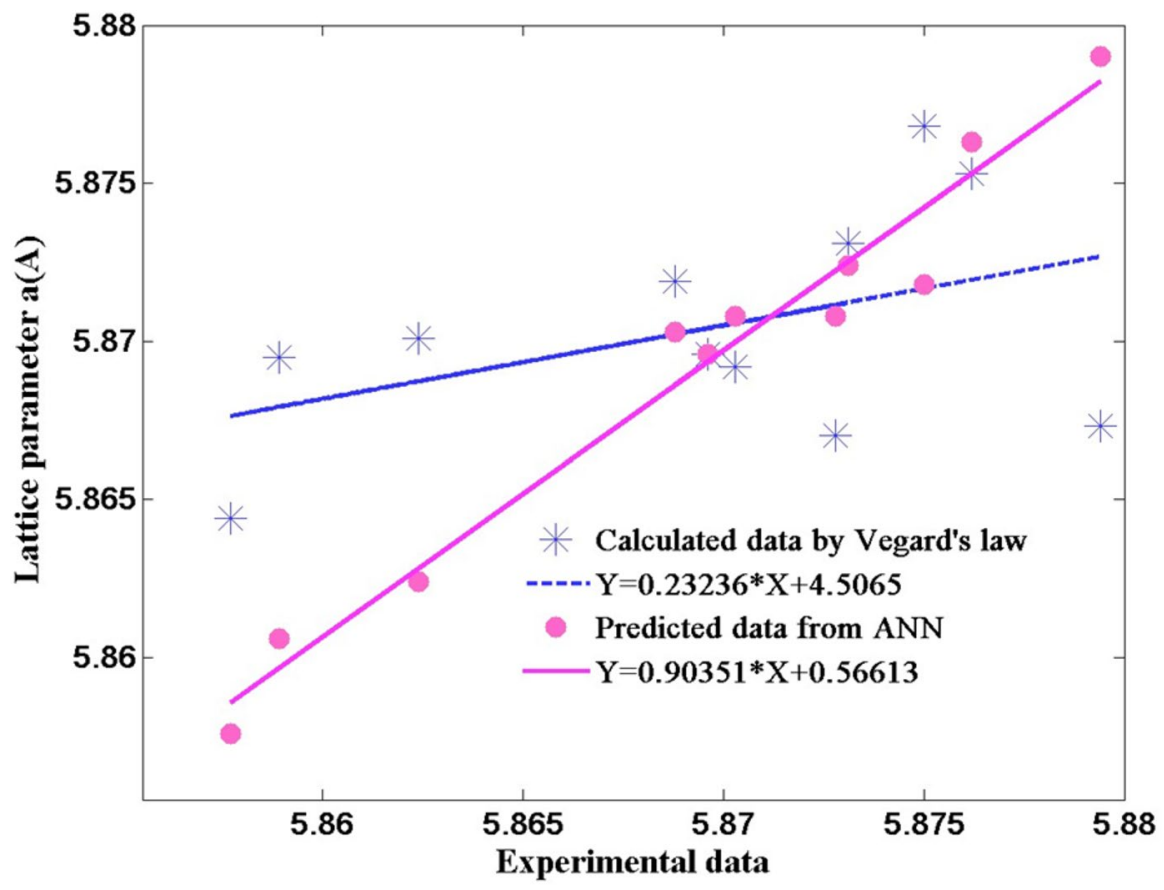

Fig. 5 Correlation between the estimated data of the lattice parameter $\mathrm{a}\left(\operatorname{In}_{1-\mathrm{x}} \mathrm{Ga}_{\mathrm{x}} \mathrm{As}_{\mathrm{y}} \mathrm{P}_{1-\mathrm{y}}\right)$ and the measured data by: The ANN-LM method (a), and the Vegard's law (Eq. 4). The adjustment of the points for each case is represented by the function $\mathrm{Y}$

\subsection{Energy gap of the quaternary $/ n_{1-x} G a_{x} A s_{y} P_{1-y} / \ln P$}

The optimal $A N N-L M$ topology for estimating energy gap $\operatorname{Eg}(x, y)$ as a function of composition $x$ and $y$ comprises an input layer with two neurons $(x, y)$, an output layer that displays gap energy, and two hidden layers that quickly and intelligently calculate the complex interconnections of neurons.

This topology was selected from several recombinations formed, based on the maximization of $R^{2}$ and the minimization of RMSE. Table 4 allows selecting the optimal transfer function, and Table 5 allows selecting the optimal number of neurons.

On the other hand, the decrease in the energy gap by increasing the $y$ composition, as clearly seen in Fig. 7, is caused by the progressive and regular replacement of Phosphorus atom sites $A s-G a-P$, by Arsenic atom sites $A s-G a-A s$. The electronic configuration of the Arsenic is $4 s^{2} 4 p^{3}$, and for the Phosphorus is $3 s^{2} 3 p^{3}$, which implies an increase in the atomic radius $\left(R_{P}(\AA)<R_{A s}(\AA)\right)$, and the decrease of the ionization energy and the electronegativity $\chi(\mathrm{P})=2.19$ and $\chi(\mathrm{As})=2.18$ according to Pauling scale. These parameters are responsible for changing the charge transfer mechanism in the material.

Figure 7a shows the incorporation of most experimental data with the data found by $A N N-L M$ model. The energies gap with the two compositions $\operatorname{Eg}(x, y)$ calculated by the Eq. (10) are further away from the target data as shown in Fig. 7b and 7c. The values of the RMSE error indicator of the $A N N-L M$ network are small compared to those generated from the data computed by Eq. (10) $\left(R M S E_{A N N}=0.0112 \operatorname{andRMSE_{(EQ10)}}=0.0341\right)$. 


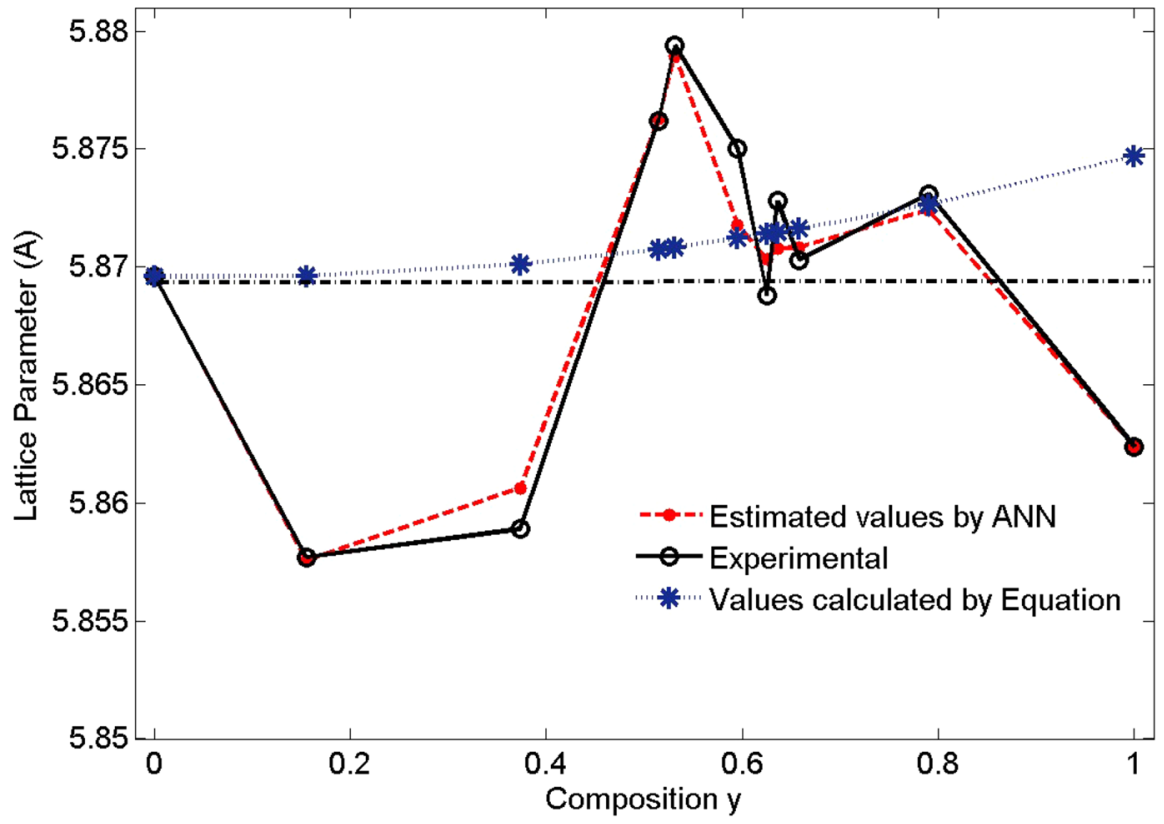

Fig. 6 Lattice parameter versus the composition y for the InGaAsP alloy adapted to InP. The circles are the experimental measurements (Nahory et al. 1978), the stars in blue show the values calculated by empirical Eq. (7), and the red points show the values predicted by ANN-LM

Table 4 Procedure for selecting the optimal transfer function for predicting the energy gap by the ANN-LM method

\begin{tabular}{|c|c|c|c|c|}
\hline $\begin{array}{l}\text { Number } \\
\text { of layers }\end{array}$ & Train function & $\begin{array}{l}\text { Number of } \\
\text { neurons }\end{array}$ & Transfer function & RMSE \\
\hline \multirow[t]{9}{*}{2} & \multirow[t]{9}{*}{ Trainlm } & \multirow[t]{9}{*}{10} & LogSig/LogSig & 0.1375 \\
\hline & & & LogSig/Purelin & 0.0149 \\
\hline & & & LogSig/TangSig & 0.0332 \\
\hline & & & Purelin/LogSig & 0.138 \\
\hline & & & Purelin/Purelin & 0.0124 \\
\hline & & & Purelin/TangSig & 0.0173 \\
\hline & & & TangSig/LogSig & 0.138 \\
\hline & & & TangSig/Purelin & 0.138 \\
\hline & & & TangSig/TangSig & 0.1398 \\
\hline
\end{tabular}

Bold values indicate the transfer function that gave the smallest RMSE

\begin{tabular}{|c|c|c|c|c|}
\hline $\begin{array}{l}\text { Number } \\
\text { of layers }\end{array}$ & Train function & Transfer Function & $\begin{array}{l}\text { Number of } \\
\text { Neurons }\end{array}$ & RMSE \\
\hline \multirow[t]{6}{*}{2} & Trainlm & Purelin/Purelin & 5 & 0.0121 \\
\hline & & & 10 & 0.0124 \\
\hline & & & 15 & 0.0117 \\
\hline & & & 20 & 0.0112 \\
\hline & & & 25 & 0.0119 \\
\hline & & & 30 & 0.0121 \\
\hline
\end{tabular}

Table 5 Procedure for selecting the optimal number of neurons with the best transfer function Purlin/Purlin for ANN-LM training 
Likewise, the coefficient of determination was estimated at $99.32 \%$ for the $A N N-L M$ model, it is higher than the coefficient of determination of the data calculated by Eq. (10), which is equal to $93.74 \%$, and which indicates an interesting deviation between the values calculated by the Eqs. (10) and the target data. This comparative study shows that $A N N-L M$ is a powerful and preeminent tool for modeling nonlinear processes such as energy gap that vary with $x$ and $y$.

The simulation results (predicted by $A N N-L M$ and targets) were described by the dispersion diagram in Fig. 8, the prediction is good because the correlation equation shows a mediocre difference (less than 0.008) between the predicted data by $A N N-L M$ and the experimental values, this confirms the results of the error indicators.

The empirical fit of the experimental curve gives the relation (11) which expresses the energy gap $E g(y)$ as a function of a single variable $y$. The $R M S E$ value in these conditions decreased strongly $R M S E_{(E Q 11)}=0.0113$, and the coefficient of determination $R_{(\mathrm{EQ} 11)}^{2}=99.31 \%$ approaches 1 , which means that the deviation is small between the data computed by Eq. (11) and the target data.

Despite this improvement which does not take the composition $x$ into consideration, the precision of the $A N N-L M$ model remains the strongest. In addition, it is robust thanks to its capacity to learn via the two experimental inputs (composition of $x$ and $y$ ), without recourse to approximations and considerations that eliminates composition. It is a powerful tool that intelligently binds input parameters and output parameters (energy gap), with the existence or absence of mismatch, and it does not require a mathematical model or a gnarled calculation. Also, it is characterized by its capacity of generalization which increases with examples of enriched and numerous training which enclose various situations. The well-formed and validated $A N N-L M$ model gives an overall response even if the impact or sensitivity of an entry is not physically significant.

\subsection{Variation of the energy gap of the quaternary $/ n_{1-x} G a_{x} A s_{y} P_{1-y} / \operatorname{In} P$ with the temperature $T$ and the composition $x$ and $y$}

The optimized $A N N-L M$ structure is selected based on the R-square and RMSE error indicators, we found that the best model among several combinations possesses a very small $R M S E\left(R M S E_{(A N N)}=7.4744 \times 10^{-4}\right)$, and the $R^{2}$ is very close to $1\left(R_{(\mathrm{ANN})}^{2} \simeq 100 \%\right)$. The architecture of the $A N N-L M$ model contains four layers, the first one is the input layer, it contains 3 variables: the composition of $x, y$ and the temperature $T$. The last one is the output layer that configures the energy gap, and between these two layers, there are two hidden layers. The optimal number of neurons is 5 and the most suitable transfer function is LogSig/Purelin(Fig. 9).

Figure 10 shows the performance of the ANN-LM model, which details the correlation between the experimental training, test and validation measurements and the estimated values by $A N N-L M$ (Fig. 10a). It should be noted that the test and the validation measures are not used in the training process. The more precise the estimated data, the closer the points are to the bisector $y=x$, and the closer the indicator $R$ approaches 1 . $(R=1)$ indicates that the training process is perfect. For the test, the correlation coefficient takes a maximum value $(R=1)$, which shows a strong linear relationship between the targets and the estimated values. The mean squared error (MSE) is determined at epoch 1 by $\left(M S E=8.4832 \times 10^{-7}\right)$, it describes the best validation of the model ANN-LM and confirms that the proposed topology has a very good performance, it is also able to predict the responses of layer output with amazing precision (Fig. 10b). 
Fig. 7 The energy gap versus the composition for the $\operatorname{In}_{1-x} \mathrm{Ga}_{\mathrm{x}} \mathrm{As}_{\mathrm{y}} \mathrm{P}_{1-\mathrm{s}}$ alloy adapted to InP. a, b Show the measures predicted by ANN-LM and the experimental data (Nahory et al. 1978) above and below the three-dimensional representation of the band gap area $\mathrm{E}(\mathrm{x}, \mathrm{y})$ of the quaternary $\operatorname{In}_{1-x} G a_{x} A s_{y} P_{1-y}$ plotted by Eq. (10). c Represents the energy gap $\mathrm{Eg}(\mathrm{y})$ versus composition y. The circles are the experimental measurements, the stars in blue show the values calculated by Eq. (10), and the red points show the values predicted by ANN-LM

In the case of the alloy $\operatorname{In}_{0.73} G a_{0.27} A s_{0.59} P_{0.41}$, the energy gap as a function of the temperature was calculated by the theoretical Eq. (14), with: $E_{g 0}=1.041 \mathrm{eV}, \alpha=7.510^{-4} \mathrm{eV}$, $\beta=611$ (Madelon and Dore 1981). The experimental values, estimated data by ANN-LM and calculated data by the theoretical Eq. (14) are shown in Fig. 11.

The comparison of the error indicators calculated from the theoretical data $\left(R M S E_{(E Q 14)}=0.0022, R_{(E O 14)}^{2}=99.08 \%\right)$, and those calculated based on the data predicted by ANN-LM $\left(R M S E_{(A N N)}=7.4710^{-4}, R_{(A N N)}^{2} \approx 99.9 \%\right)$ shows the power of the ANN-LM tool.

In addition, using the theoretical equation requires a calculation to extract the values of $\alpha, \beta$ and $E_{g 0}$ at each variation of composition $x$ or $y$, which makes the physical process complicated, and the analytical model is not unique. This problem is solved by the model $A N N-L M$ thanks to the weights which connects between the input parameters $(x, y, T)$ and the output parameter $(E g)$.

On the other hand, the estimate of the energy gap in the absence of the pressure $E_{g}(T, p=0)$ makes it possible to minimize the error of the calculation of the energy gap of the material subjected to the effect of the pressure $\mathrm{E}_{\mathrm{g}}(\mathrm{T}, \mathrm{p})$ using the relation the empirical relation (20).

\subsection{Variation of the energy gap of the quaternary $\ln _{1-x} G a_{x} A s_{y} P_{1-y} / \operatorname{In} P$ with the temperature and the pressure}

The architecture of the best structure $A N N-L M$ found is represented in Fig. 2a, it contains four layers: an input layer with two variables (the temperature $T$ and the pressure $P$ ). An output estimates the energy gap, and between these two layers, there are two hidden layers. The number of neurons is 20, the activation function used is TanSig/Purelin (Fig. 12b).

We notice that the energy gap $E_{g}^{\Gamma}$ of binary alloys $G a A s$ at $T=300 \mathrm{~K}$ increases with increasing pressure. this means that the energy band for $\Gamma$-point is moved upwards. On the other hand, as shows the Fig. 13, the experimental measurements, the data estimated by $A N N-L M$ and the data calculated by the Eq. (20) in the interval [0-90 Kbar] are all in agreement. The $R^{2}$ is almost equal to 1 , and the error RMSE of the two estimates is very small $\left(R M S E_{(E Q 20)}=6.8710^{-5}, R M S E_{(A N N)}=9.4610^{-4}\right)$. The ANN-LM method does not require a previous knowledge of the parameters that constitute the empirical equation, and which are the energy band gap at zero pressure $E_{g}(T, p=0)^{d, i d}$, and the pressure coefficients $($ bandc).

\section{Conclusion}

In this work, we used the Artificial Neural Networks ANN, to study the physical properties of the quaternary $\operatorname{In}_{1-x} G a_{x} A s_{y} P_{1-y} / \operatorname{InP}$ with $0 \leq \mathrm{x} \leq 0.47$ and $0 \leq \mathrm{y} \leq 1$. Learning was optimized by the Levenberg Maquardt backpropagation algorithm. The $A N N-L M$ hybrid 

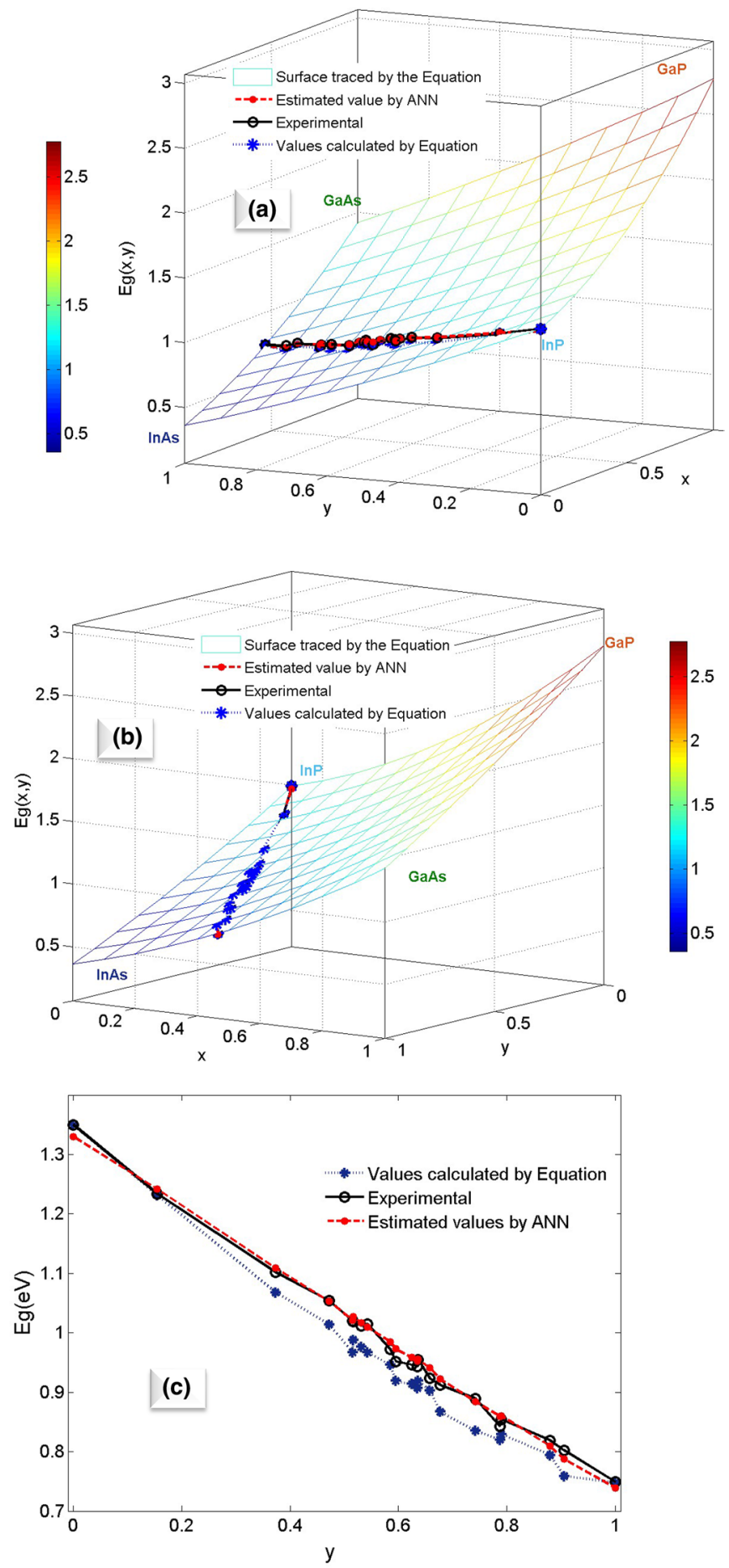


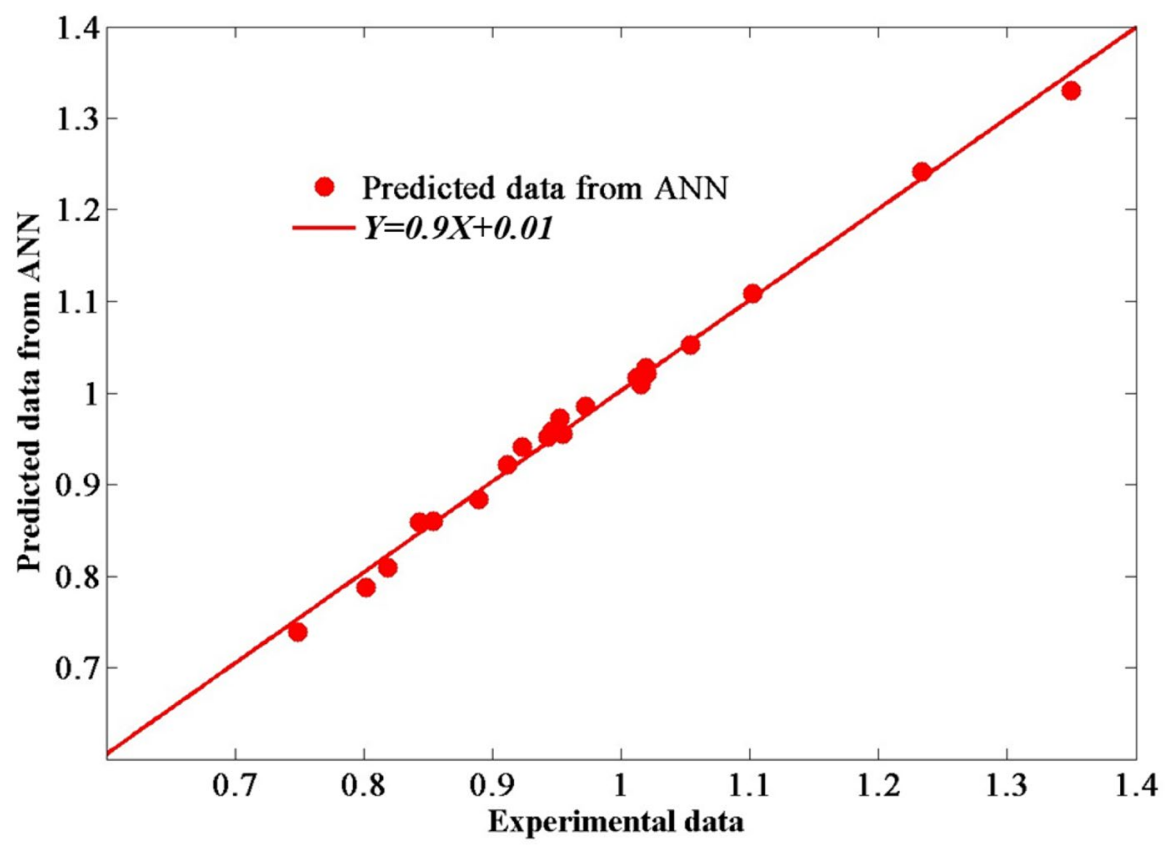

Fig. 8 Correlation between the measured and estimated data by ANN-LM of the energy gap. The adjustment of the points is represented by the function $\mathrm{Y}$

system was used to determine optimal combinations capable of predicting the lattice parameter ' $a$ ' and the band-gap energy at different $x$, and $y$, also the band-gap energy at different $x, y$, and temperature, and finally the band-gap energy at different temperatures and 


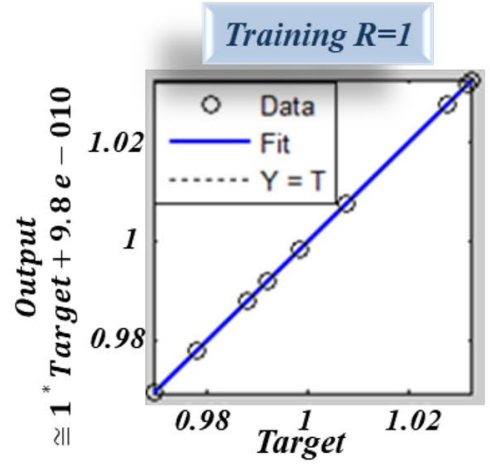

\section{Test $R=1$}

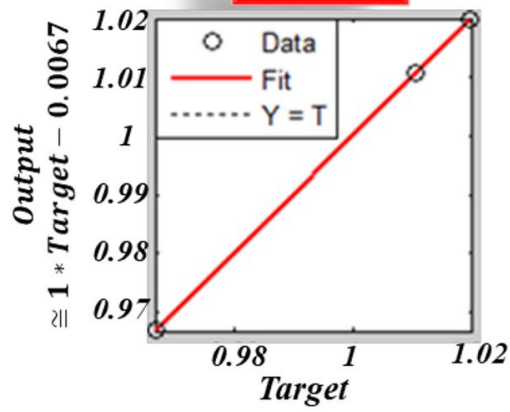

Validation $R=0.99998$

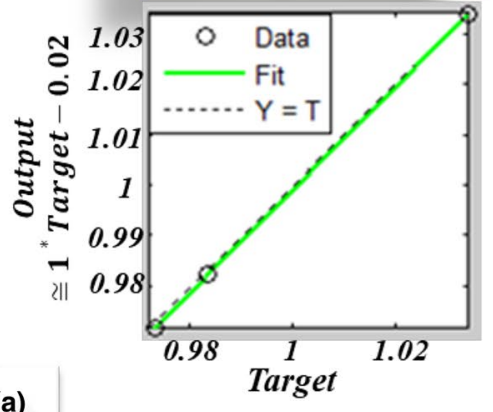

(a)

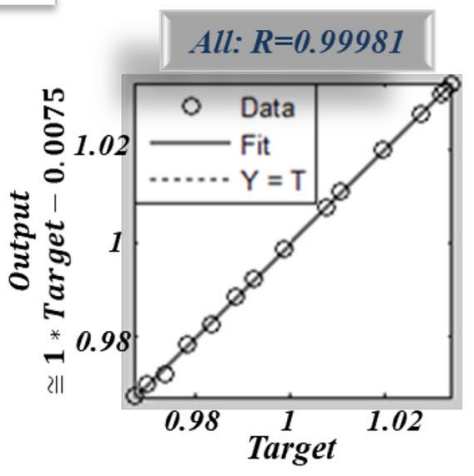

\section{Best Validation Performance is $8.4832 e-007$ at epoch 1}

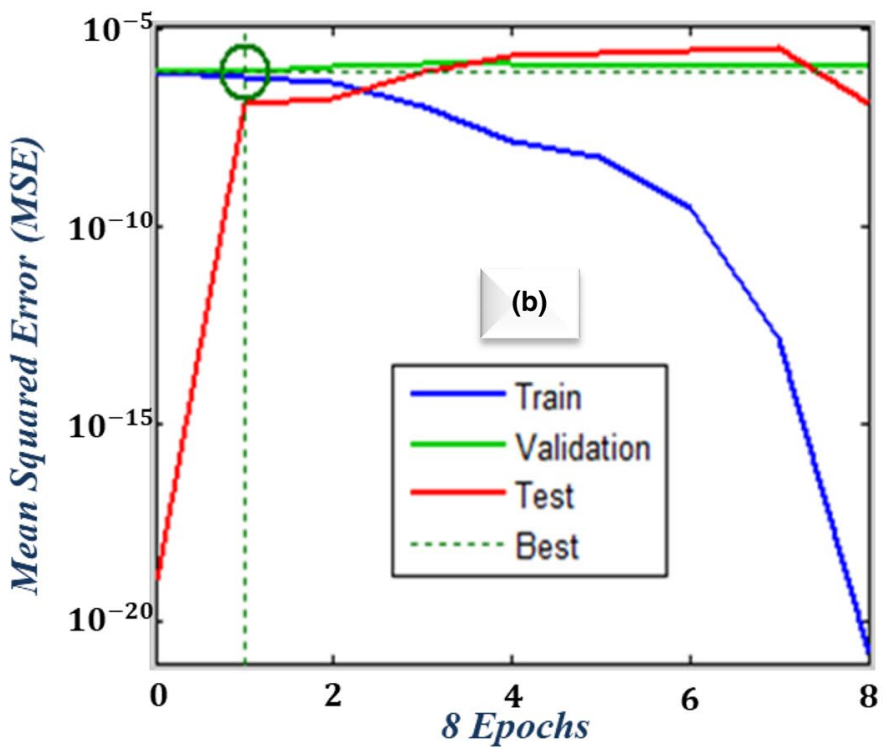

Fig. 9 Diagram of neural network for identification $\operatorname{Eg}(\mathrm{x}, \mathrm{y}, \mathrm{T})$. 


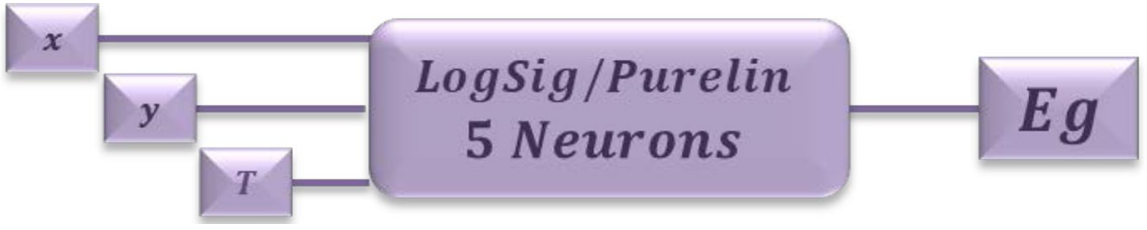

Fig. 10 Effectiveness of the neural network: (a) Correlation between target data (Eg) and the estimated output data by ANN-LM. (b) The procedure for training the ANN-LM through three stages: Testing, validation and train

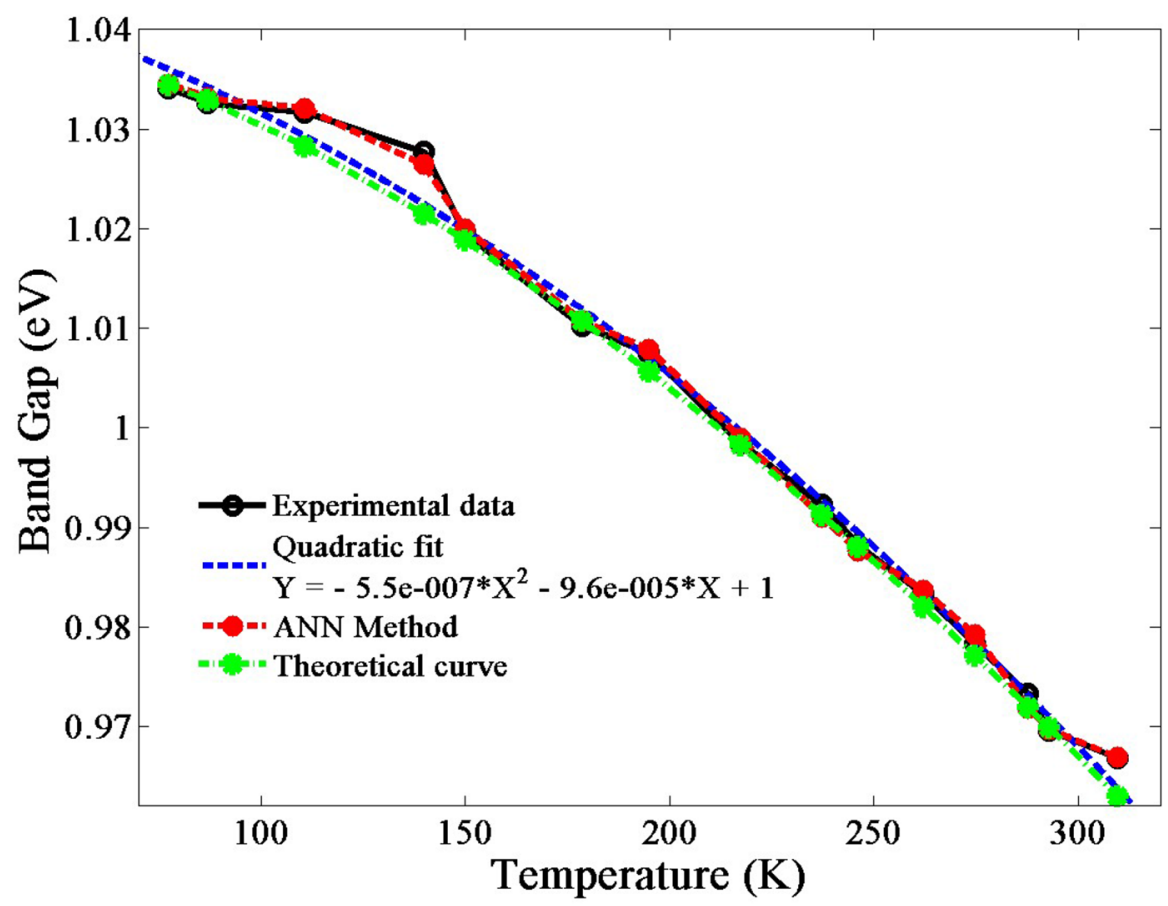

Fig. 11 Comparison between the experimental values of $\mathrm{Eg}(\mathrm{T})$ (Madelon and Dore 1981), the calculated data by the theoretical semi-empirical curve (Eq. 14) and the estimated data by ANN-LM of the quaternary $\mathrm{In}_{0.73} \mathrm{Ga}_{0.27} \mathrm{As}_{0.59} \mathrm{P}_{0.41}$ 


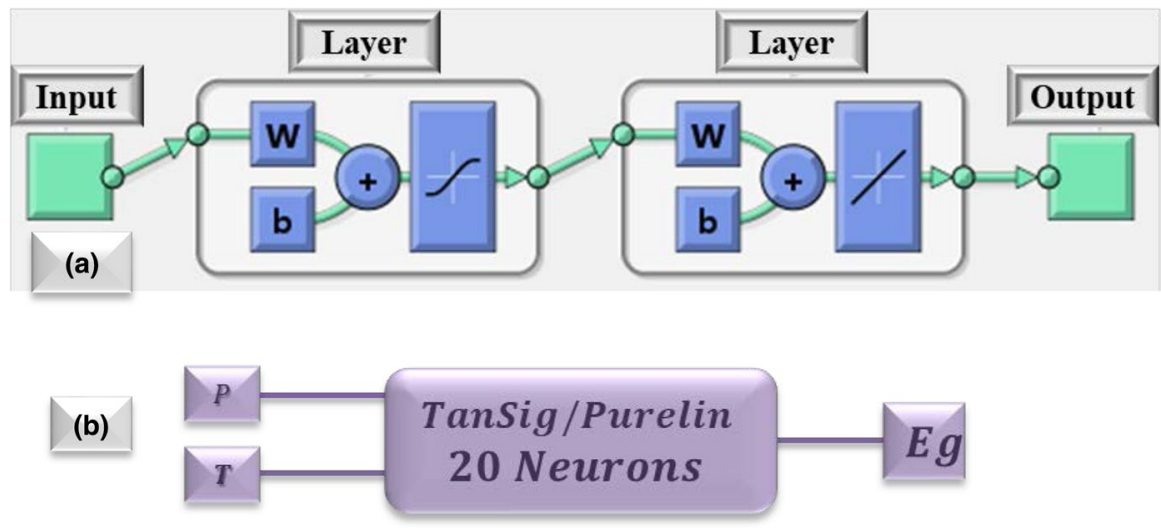

Fig. 12 Diagram of neural network for identification of $\operatorname{Eg}(\mathrm{T}, \mathrm{P})$ : $\mathbf{a}$ describes the number of layers, and $\mathbf{b}$ describes the activation function, the number of neurons, the inputs and the output of the ANN-LM

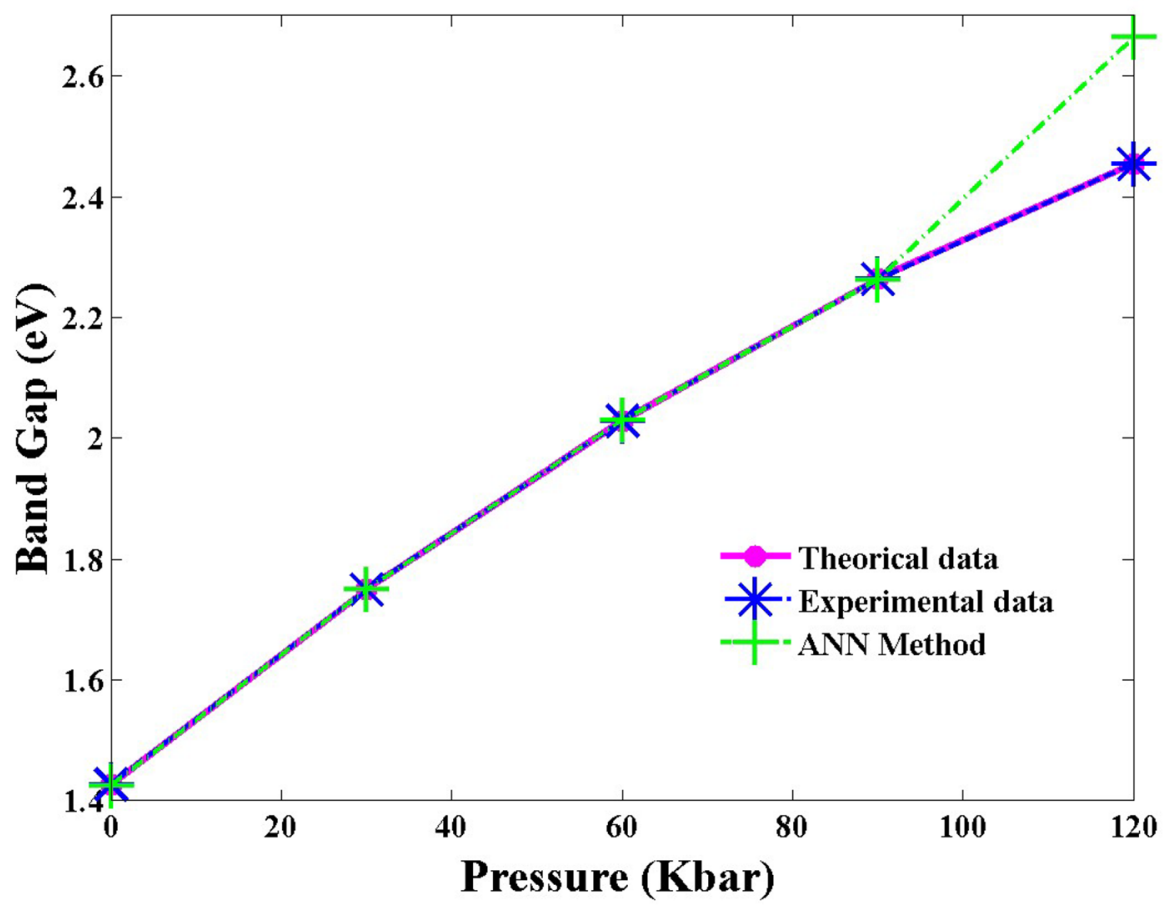

Fig. 13 The energy band gap $\mathrm{E}_{\mathrm{g}}^{I^{\prime}}$ of binary alloys GaAs versus pressure at room temperature: The points in pink are the values calculated by the Eq. (20), the stars in blue are the experimental data (Elabsy et al. 2010), and the marks in green are the values estimated by ANN-LM

pressures. Performance indicators $\left(R^{2}\right)$ and $R M S E$ have shown that the four ANN-LM networks proposed are reliable and robust. The coefficient of determination $\left(R^{2}\right)$ of the optimal recombinations exceeded $95.84 \%$, and the RMSE error was minimized, it does not exceed 
0.0014. This shows that the experimental and the prediction data are in excellent agreement. The dispersion diagrams confirm the effectiveness of the models, their outputs show good compliance with experimental data. On the other hand, there is a considerable difference between experimental data of physical properties $a(x, y), E g(x, y, T)$ and the results of proposed empirical equations derived from interpolation. The high predictive capacities of the $A N N-L M$ are obtained without any approximation to eliminate the variable $\boldsymbol{x}$ by considering the perfect match between the material and the substrate. On the other hand, the calculation of the refractive index of the alloy becomes easy to calculate using values estimated by $A N N-L M$. This method can be used to control and estimate the energy gap variation of the photovoltaic cell subjected to different temperatures.

Acknowledgements The author Amal Tarbi would like to acknowledge distinguished professor B. Oukarfi for their valuable scientific discussions and recommendations.

Open Access This article is distributed under the terms of the Creative Commons Attribution 4.0 International License (http://creativecommons.org/licenses/by/4.0/), which permits unrestricted use, distribution, and reproduction in any medium, provided you give appropriate credit to the original author(s) and the source, provide a link to the Creative Commons license, and indicate if changes were made.

\section{References}

Adachi, S.: Material parameters of In1-x Ga x As y P1-y and related binaries. J. Appl. Phys. 53(12), 8775-8792 (1982)

Afram, A., Janabi-Sharifi, F., Fung, A.S., Raahemifar, K.: Artificial neural network (ANN) based model predictive control (MPC) and optimization of HVAC systems: A state of the art review and case study of a residential HVAC system. Energy Build. 141, 96-113 (2017)

Agrawal, G.P., Dutta, N.K.: Long-wavelength semiconductor lasers. Van Nostrand Reinhold, New York (1986)

Aish, A.M., Zaqoot, H.A., Abdeljawad, S.M., Samaher, M.: Artificial neural network approach for predicting reverse osmosis desalination plants performance in the Gaza Strip. Desalination 367, 240-247 (2015)

Akiba, S., Sakai, K., Matsushima, Y., Yamamoto, T.: Room temperature cw operation of InGaAsP/InP heterostructure lasers emitting at $1.56 \mu \mathrm{m}$. Electron. Lett 15, 606-607 (1979)

Chen, Z., Ma, W., Wei, K., Wu, J., Li, S., Xie, K., Lv, G.: Artificial neural network modeling for evaluating the power consumption of silicon production in submerged arc furnaces. Appl. Therm. Eng. 112, 226-236 (2017)

Degheidy , A.R., Elkenany, E.B.: Temperature and pressure dependence of the electronic and optical properties of GaxIn1 - xAsyP1 - y matching different substrates. Physica B 456, 213-220 (2015)

Dkhichi, F., Oukarfi, B., Fakkar, A., Belbounaguia, N.: Parameter identification of solar cell model using Levenberg-Marquardt algorithm combined with simulated annealing. Sol. Energy 110, 781-788 (2014)

Dkhichi , F., Oukarfi, B., Kouari, Y.E., Ouoba, D., Fakkar, A.: Neural network based integration of MPPT and diagnosis of degradation for photovoltaic module. Opt. Quant. Electron. 48, 105 (2016)

Elabsy, A.M., Degheidy, A.R., Abdelwahed, H.G., Elkenany, E.B.: Pressure response to electronic structures of bulk semiconductors at room temperature. Phys. B 405, 3709-3713 (2010)

Fashinger, W., Krump, R., Brunthaler, G., Ferreira, S., Sitter, H.: Appl. Phys. Lett. 65, 3125 (1994)

Fiedler, F., Schlachetzki, A.: Optical parameters of InP-based waveguides. Solidstate Electronics 30, 73-83 (1987)

Gazzaz, N.M., Yusoff, M.K., Aris, A.Z., Juahir, H., Ramli, M.F.: Artificial neural network modeling of the water quality index for Kinta River (Malaysia) using water quality variables as predictors. Mar. Pollut. Bull. 64, 2409-2420 (2012)

Geyikçi, F., Kılıç, E., Coruhc, S., Elevli, S.: Modelling of lead adsorption from industrial sludge leachate on red mud by using RSM and ANN. Chem. Eng. J. 183, 53-59 (2012) 
Glisson, T.H., Hauser, J.R., Littlejohn, M.A., Williams, C.K.: Energy bandgap and lattice constant contours of III-V quaternary alloys. J. Electron. Mater. 7, 1-16 (1978)

Houston, P.A.: Growth and characterization of InGaAsP lattice-matched to InP. J. Mater. Sci 16, 2935-2961 (1981)

Jones, D., Lettington, A.H.: Pseudopotential calculations of the band structure of GaAs, InAs and (GaIn) as alloys. Solid State Commun. 7, 1319-1322 (1969)

Krijn, M.P.C.M.: Heterojunction band offsets and effective masses in III-V quaternary alloys. Semicond. Sci. Technol. 6, 27-31 (1991)

Kumari, A., Das, S.K., Srivastava, P.K.: Modeling Fireside Corrosion Rate in a Coal Fired Boiler Using Adaptive Neural Network Formalism. Portugaliae Electrochimica Acta 34, 23-38 (2016)

Madelon, R., Dore, M.: Derivative transmission measurements of the temperature dependance of the $\gamma-\gamma$ transition in the Ga0. 73In0. 27As0. 59P0. 41 compound. Solid State Commun. 39, 639-641 (1981)

Manasevit, H.M.: Single-crystal gallium arsenide on insulating substrates. Appl. Phys. Lett. 12, 156-159 (1968)

Mei, T.: Interpolation of quaternary III-V alloy parameters with surface bowing estimations. J. Appl. Phys. 101, 013520 (2007)

Mekki, H., Mellit, A., Salhi, H.: Artificial neural network-based modelling and fault detection of partial shaded photovoltaic modules. Simul. Model. Pract. Theory 67, 1-13 (2016)

Nahory, R.E., Pollack, M.A., Johnston Jr., W.D., Barns, R.: Band gap versus composition and demonstration of Vegard's law for In1 - x Ga x As y P1 - y lattice matched to InP. Appl. Phys. Lett. 33, 659-661 (1978)

Nakajima, K., Yamaguchi, A., Akita, K., Kotani, T.: Composition dependence of the band gaps of In $1-\mathrm{x}$ Ga x As1-y P y quaternary solids lattice matched on InP substrates. J. Appl. Phys. 49, 5944-5950 (1978)

Seifert, S., Runge , P.: Revised refractive index and absorption of $\operatorname{In}_{1-\mathrm{x}} \mathrm{Ga}_{\mathrm{x}} \mathrm{As}_{\mathrm{y}} \mathrm{P}_{1-\mathrm{y}}$ lattice-matched to $\mathrm{InP}$ in transparent and absorption IR-region. Opt. Mater. Express 6, 629-639 (2016). https://doi.org/10.1364/ ome.6.000629

Shim, K., Rabitz, H., Chang, J.H., Yao, T.: Energy band gap of the alloy Zn1-xMgxSeyTe1-y lattice matched to ZnTe, InAs and InP. J. Cryst. Growth 214, 350-354 (2000)

Tarbi, A., Mani, E.H.: Study of electroluminescent diodes of GAAS 1-XPX using conductance spectroscopy. J. Adv. Mater. Sci. Eng. Sci. 4(3), 1-7 (2015). https://doi.org/10.14810/ijamse.2015.4301

Tarbi, A., Atmani, E.H., Sellam, M.A.: Control and diagnostic of the complex impedance of selected perovskite compounds. Opt. Quant. Electron. 49, 337 (2017)

Varshni, Y.P.: Temperature dependence of the energy gap in semiconductors. Physica 34, 149-154 (1967)

Vurgaftman, I., Meyer, J.R., Ram-Mohan, L.R.: Band parameters for III-V compound semiconductors and their alloys. J. Appl. Phys. 89, 5815-5875 (2001)

Waqas, A., Alippi, A., Melati, D., \& Melloni, A.: An improved model to predict thermo-optic coefficient in InGaAsP waveguides. In: Transparent Optical Networks (ICTON), 2016 18th International Conference on, pp. 1-4. IEEE (2016)

Xuan, Y., Wu, Y.Q., Ye, P.D.: High-performance inversion-type enhancement-mode InGaAs MOSFET with maximum drain current exceeding $1 \mathrm{~A} / \mathrm{mm}$. IEEE Electron Dev. Lett. 29, 294-296 (2008)

Yamazoe , Y., Nishino, T., Hamakawa, Y., Kariya, T.: Bandgap energy of InGaAsP quaternary alloy. Jpn. J. Appl. Phys. 19, 1473-1479 (1980)

Yamazoe, Y., Nishino, T., Hamakawa, Y.: Electroreflectance study of InGaAsP quaternary alloys lattice matched to InP. IEEE J. Quant. Electron. 17, 139-144 (1981)

Yu, P.Y., Cardona, M.: Fundamentals of Semiconductors. Springer, Berlin (1996) 